\title{
Topology, holes and sources
}

\author{
Alexander Afriat
}

August 6, 2013

\begin{abstract}
The Aharonov-Bohm effect is often called "topological." But it seems no more topological than magnetostatics, electrostatics or Newton-Poisson gravity (or just about any radiation, propagation from a source). I distinguish between two senses of "topological."
\end{abstract}

\section{The Aharonov-Bohm effect}

A wavefunction is split into two, and these, having enclosed a (simply-connected) region $\omega$ containing a solenoid, are made to interfere on a screen. The enclosing wavefunction is sensitive to any enclosed electromagnetism inasmuch as the electromagnetic potential $^{1} A$, a one-form, contributes a phase

$$
\exp i \oint_{\partial \omega} A
$$

to (the wavefunction along) the boundary $\partial \omega$ and hence to the interference pattern on the screen. The electromagnetism on $\omega$ is related to the circulation around the boundary by Stokes' theorem

$$
C=\oint_{\partial \omega} A=\iint_{\omega} d A
$$

The electromagnetic field ${ }^{2} F=d A$ produced by the solenoid is circumscribed to a middle region $\lambda \subset \omega$ surrounded by an isolating region ${ }^{3} \lambda^{\prime}=\omega-\lambda$ where $F$ vanishes but not $A$. The full Aharonov-Bohm effect $t^{4}$ can be considered the 'differential' or 'incremental' sensitivity of the interference pattern to variations in the current through the solenoid. ${ }^{5}$

\footnotetext{
${ }^{1}$ By "potential" I just mean primitive: the potential of the electromagnetic two-form $F=d A$ is its primitive $A \leftrightarrow(\mathbf{A}, \varphi)$, the potential of the magnetic two-form $\mathbf{B}=d \mathbf{A}$ is its primitive $\mathbf{A}$, the potential of the electric one-form $* \mathbf{E}=d \varphi$ is its primitive $\varphi$ (the Hodge dual $*$ being taken in three dimensions), the potential of the three-form density $\rho=d \mathbf{E}$ is its primitive $\mathbf{E}$.

${ }^{2}$ It is perhaps easiest to think of $F$ as a purely magnetic field $\mathbf{B}$ produced by the current density $\mathbf{J}=d * \mathbf{B}$ in the solenoid.

${ }^{3}$ It will be convenient to view $\lambda$ and $\omega$ as concentric disks.

${ }^{4}$ Ehrenberg \& Siday (1949), Aharonov \& Bohm (1959)

${ }^{5} C f$. Batterman (2003, p. 555): "Similarly, in the AB effect, it appears that we will need to refer to different nonseparable holonomy properties for each case in which there is a different flux running through the solenoid."
} 


\section{The topological interpretation $_{1}$}

I will distinguish between two (related but) different senses of "topological":

1. Topological 1 : related to the presence of a hole (containing a source, a solenoid or charge for instance).

2. Topological 2 : invariant under appropriate continuous deformations.

The topological $_{1}$ interpretation $^{6}$ of the Aharonov-Bohm effect can be formulated as follows: If $A$ were closed throughout a simply-connected region $\omega$ it would also be

\footnotetext{
${ }^{6}$ Aharonov \& Bohm (1959, p. 490): "in a field-free multiply-connected region of space, the physical properties of the system still depend on the potentials." Wu \& Yang (1975, p. 3845): "The famous BohmAharonov experiment [...] showed that in a multiply connected region where $f_{\mu \nu}=0$ everywhere there are physical experiments for which the outcome depends on the loop integral [...] around an unshrinkable loop." And p. 3856: " $f_{\mu \nu}$ underdescribes electromagnetism because of the Bohm-Aharonov experiment which involves a doubly connected space region." Nash \& Sen (1983, p. 301): "We [...] consider the consequence of assuming the field $\mathbf{F}$ to be identically zero in some region $\Omega$. At first one may think that there will be no physically measurable electromagnetic effects in such a region $\Omega$. This is not so, effects may arise if the topology of $\Omega$ is non-trivial, e.g. if $\Omega$ is not simply connected. [...] In terms of parallel transport one says that zero curvature does not imply trivial parallel transport if the region in which the curvature is zero is not simply connected. This underlies the fact that there is a sense in which the connection is a more fundamental object than the curvature, even though a connection is gauge dependent and not directly measurable." Ryder (1996, p. 101-4): "the Bohm-Aharonov effect owes its existence to the non-trivial topology of the vacuum [...]. The Bohm-Aharonov effect is the simplest illustration of the importance of topology in this branch of physics. [...] The relevant space in this problem is the space of the vacuum, i.e. the space outside the solenoid, and that space is not simply connected. [...] It is thus an essential condition for the Bohm-Aharonov effect to occur that the configuration space of the vacuum is not simply connected. $[\ldots]$ in other words, it is because the gauge group of electromagnetism, $U_{1}$, is not simply connected that the Bohm-Aharonov effect is possible. [...] The configuration space of the Bohm-Aharonov experiment is the plane $\mathbb{R}^{2}[\ldots]$ with a hole in, and this is, topologically, the direct product of the line $\mathbb{R}^{1}$ and the circle $[. .$.$] . There is, nevertheless, a positive effect on the interference fringes. The mathematical reason for$ this is that the configuration space of the null field (vacuum) is the plane with a hole in [...]." Batterman (2003, p. 544): "We now have a $U(1)$ bundle over a nonsimply connected base space: $\mathbb{R}^{2}-\{$ origin $\}$. This fact is responsible for the AB effect." Ibid. pp. 552-3: "most discussions of the AB effect very quickly idealize the solenoid to an infinite line in space or spacetime. The flux, in this idealization, just is the abstract topological property of having space or spacetime be nonsimply connected. [...] The issue is whether the idealizations - [...] and nonsimply connected space in the AB effect-do better explanatory work than some less idealized description. I believe that the idealized descriptions do, in fact, do a better job." Ibid. p. 554: "It seems to me that for a full understanding of these anholonomies, one needs to appeal to the topology and geometry of the base space. [...] If we take seriously the idea that topological features of various spaces $[\ldots]$ can play an explanatory role [...]." Footnote 29, same page: "it is most fruitful to treat the $\mathrm{AB}$ solenoid as an idealization that results in the multiple connectedness of the base space of a fiber bundle." Ibid. p. 555: "The different cases are unified by the topological idealization of the solenoid as a string absent from spacetime which renders spacetime nonsimply connected. [...] This topological feature enables us to understand the common behaviour in different $\mathrm{AB}$ experiments [...]. [...] how can it possibly be the case that appeal to an idealization such as the $\mathrm{AB}$ solenoid as a line missing from spacetime, provides a better explanation of genuine physical phenomena than can a less idealized, more "realistic" account where one does not idealize so severely? [ ...] quite often [...] appeal to highly idealized models does, in fact, provide better explanations." Martin (2003, p. 48): "in the case of non-trivial spatial topologies, the gauge-invariant interpretation runs into potential complications. [...] So-called holonomies [...] encode physically significant information about the global features of the gauge field." See also Lyre (2001, pp. S377-80), Nounou (2003), Lyre (2004, p. 659), Agricola \& Friedrich (2010, p. 275).
} 
exact, and hence expressible as the gradient $A=d \mu$ of a zero-form $\mu$; the flux

$$
\oint_{\partial \omega} d \mu=\iint_{\omega} d^{2} \mu
$$

through the boundary $\partial \omega$ would then vanish, as $d^{2}$ does. But here $A$ is closed on $\lambda^{\prime}$; from $d A=\left.0\right|_{\lambda^{\prime}}$ it does not follow that $A$ is exact, nor that the flux through the enclosing loop vanishes: it may or may not.

The existence of the source responsible for the effect is therefore ruled out by one topology ( $A$ closed throughout a simply-connected region) but not another.

The same applies to a simply-connected three-dimensional region $\Omega$ enclosed by a two-dimensional boundary $\partial \Omega$. If the two-form $\mathbf{E}$ were closed throughout $\Omega$ it would also be exact, and hence expressible as the curl $\mathbf{E}=d \zeta$ of a one-form $\zeta$; the flux

$$
\iint_{\partial \Omega} d \zeta=\iiint_{\Omega} d^{2} \zeta
$$

through the boundary would then vanish. But if the region on which $\mathbf{E}$ is closed has a hole in it, the flux through the enclosing surface may or may not vanish.

This is precisely what we have in electrostatics, where the electric field $\mathbf{E}=* d \varphi$ is (Hodge-dual to) the gradient $d \varphi$ of the scalar potential $\varphi$. The vanishing divergence $d \mathbf{E}$ expresses the conservation of electricity where none is created, away from the charges that produce $\mathbf{E}$ according to the Maxwell-Poisson equation $d \mathbf{E}=d * d \varphi=\rho$, $\rho$ being the charge density. If the divergence $d \mathbf{E}$ vanished throughout the volume $\Omega$, there would be no electricity produced and hence none radiated through the enclosing surface. ${ }^{7}$ But a charge in $\Omega$ - say in a region ${ }^{8} \Lambda \subset \Omega$ isolated by $\Lambda^{\prime}=\Omega-\Lambda$ —would prevent electricity from being conserved throughout $\Omega$.

We have the same formalism in Newton-Poisson gravity, where $\varphi$ is the gravitational potential, $d \varphi$ and $\mathbf{E}$ both represent gravitational force, and $\rho$ is the mass density. Gravity $^{9}$ would therefore be another topological ${ }_{1}$ effect.

Again, the topology of the region where the 'potential' ( $A$ or $\mathbf{E}$ or whatever) is closed tells us relatively little: if the region were simply-connected, conservation would be general within the enclosing surface since there could be no holes containing sources; and if nothing were created inside the enclosing surface, the total radiation through it would vanish. But if the topology does not allow the presence of holes to be ruled out, the presence of sources in them cannot either; and sources would produce a flux through the enclosing surface.

A non-trivial topology cannot, on its own at any rate, rule out the absence of a source either. Nor does it provide the 'amount' or 'intensity' of the possible source (which would tell us the intensity of the effect - the flux through the enclosing surface). So the full Aharonov-Bohm effect, which can be considered 'incremental,' is hardly topological ${ }_{1}$, or at any rate no more so than electrostatics or Newton-Poisson gravity.

The implications

$$
\left(\left[d A=\left.0\right|_{\omega}\right] \&[\omega \text { simply-connected }]\right) \Rightarrow[A \text { exact }] \Rightarrow\left[\oint_{\partial \omega} A=0\right]
$$

\footnotetext{
${ }^{7}$ Over and above any divergence-free electrical background that may or may not be present.

${ }^{8}$ It will be convenient to view $\Lambda$ and $\Omega$ as concentric spheres.

${ }^{9}$ Or rather the total gravitational attraction radiated by a mass.
} 
are legitimate, unlike

$$
\left(\left[d A=\left.0\right|_{\omega}\right] \& \neg[\omega \text { simply-connected }]\right) \Rightarrow \neg[A \text { exact }] \Rightarrow \neg\left[\oint_{\partial \omega} A=0\right],
$$

or

$$
\left(\left[d A=\left.0\right|_{\omega}\right] \&[\omega \text { multiply-connected }]\right) \Rightarrow[A \text { not exact }] \Rightarrow\left[\oint_{\partial \omega} A \neq 0\right] .
$$

\section{Topological 2 effects}

\subsection{Aharonov-Bohm}

The Aharonov-Bohm effect is topological 2 in the sense that certain basic quantities (say the circulation $C$ and resulting interference pattern) are invariant under appropriate continuous deformations; but it seems that (fundamental aspects of) electrostatics and Newton-Poisson gravity are just as topological 2 . Magnetostatics may be even more topological $_{2}$.

Since one measures the curvature $F=d A=d A^{\prime}$ rather than $A$ itself, the freedom expressed by the substitution

$$
A \mapsto A^{\prime}=A+d \xi
$$

( $\xi$ being a zero-form) is invisible. Such transformations deform the level sets of $A$ 's local potential ${ }^{10} \gamma$. One can first imagine a purely 'radial' or 'angular' $\gamma$ (with values running from zero to $2 \pi k=C)$, ${ }^{11}$ whose level lines are straight rays radiating through the annulus $\lambda^{\prime}$ from the inner disk $\lambda$ to the edge $\partial \omega$. A gauge transformation (2) would then deform the level rays, bending them without making them cross. The circle $\partial \omega$ can likewise be deformed into any loop going around the solenoid once. The AharonovBohm effect is topological $_{2}$ in the sense that neither deformation affects the circulation $C$ (or the resulting interference pattern).

It is perhaps easier to picture the denumerable set $\left\{\gamma_{1}, \ldots, \gamma_{N}\right\}$ of level curves at intervals of $C / N$ than all of them; they will each be cut once ${ }^{12}$ by any loop going around the solenoid once. ${ }^{13}$

\footnotetext{
${ }^{10}$ For wherever $A$ is closed it can be written locally as the gradient $A=d \gamma$ of a zero-form $\gamma$-just as $\mathbf{E}$ can be written locally, wherever it is closed, as the curl $\mathbf{E}=d \zeta$ of a one-form $\zeta$.

${ }^{11}$ Such a $\gamma$ cannot be continuous everywhere; we can imagine a single discontinuity, say on the ray with values $\gamma=2 \pi n k$, where the integer $n$ is zero then one, $k=C / 2 \pi$ being a constant.

${ }^{12}$ One should really say an odd number of times, as Jean-Philippe Nicolas has pointed out to me. Crossings in opposite directions cancel, and add nothing to the integral.

${ }^{13}$ Batterman (2003, pp. 545-6): "The phase or anholonomy depends continuously on the flux in the solenoid, but $[\ldots]$ it depends discontinuously upon the shape of the circuit. For example, two loops around gives an anholonomy twice that of one loop around for constant magnetic flux." And p. 555: "The different cases are unified by the topological idealization of the solenoid as a string absent from spacetime which renders spacetime nonsimply connected. In this way we can understand why, for a given fixed magnetic flux, a loop that goes $n$ times around the solenoid will have an anholonomy that is $n$ times that of a loop that goes around once." A loop going around twice will cross each level curve twice. Alternatively, two different loops will also catch the same flux twice. Similar double-counting can be arranged in electrostatics too: a membrane enclosing the source twice, or two different membranes.
} 
Whereas here the deformations are allowed by (and part of) theory, in the next cases they will be counterfactual.

\subsection{Electrostatics}

The basic law here, the Gauß-Maxwell equation

$$
\iint_{\partial \Omega} \mathbf{E}=\iiint_{\Omega} d \mathbf{E}=\iiint_{\Omega} \rho
$$

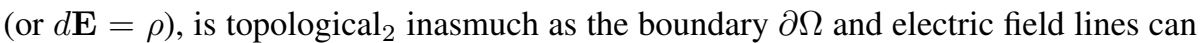
be continuously deformed without affecting the integral (3) - which corresponds to the (circulation giving rise to the) Aharonov-Bohm effect. We can imagine a spherically symmetric charge distribution $\rho$ contained in $\Lambda \subset \Omega$ (everything concentric): the electrical field lines radiated by the charge in $\Lambda$ correspond to the level rays radiating from the solenoid in $\lambda$. Nothing in electrostatics prevents the deformation of $\partial \Omega$. Admittedly the electric rays cannot be bent without violating $\mathbf{E}=* d \varphi$; despite preserving the divergence $d \mathbf{E}=d \mathbf{E}^{\prime}$, the transformation

$$
\mathbf{E} \mapsto \mathbf{E}^{\prime}=\mathbf{E}+d \alpha
$$

(the three-dimensional version of (2), $\alpha$ being a one-form) is counterfactual-which does not prevent a conditional characterisation of the effect as topological 2 : "the integral would remain the same even if the field lines were bent."14

Most of this applies, mutatis mutandis, to Newton-Poisson gravity.

Why bother with obvious facts about integration? Because much is made of the deformable loop $\partial \omega$ (which corresponds to the deformable surface $\partial \Omega$ ) and gauge transformation (2) (which corresponds to the counterfactual transformation (4)).

\subsection{Magnetostatics}

The basic law here, Mawell's equation

$$
\iint_{\partial \Omega} \mathbf{B}=\iiint_{\Omega} d \mathbf{B}=0
$$

(or $d \mathbf{B}=0$ ), holds because a magnet has two poles, that act as source and sink of the same field lines, which form loops going from one pole to the other: all magnetism produced is eventually recovered. If a magnetic loop crosses the boundary $\partial \Omega$ it will cross it again ${ }^{15}$ on the way back to the magnet, thus erasing whatever it contributed to the integral on the way out. The law is topological ${ }_{2}$ in that the boundary $\partial \Omega$ and field lines can be deformed ${ }^{16}$ continuously without affecting the integral. Nothing in magnetostatics prevents the deformation of $\partial \Omega$; the deformation of the field lines by $\mathbf{B} \mapsto \mathbf{B}^{\prime}=\mathbf{B}+d \beta$ is again counterfactual, since the magnetic field is observable.

\footnotetext{
${ }^{14}$ Here I am indebted to Dennis Dieks, Éric Gourgoulhon and Jean-Philippe Nicolas.

${ }^{15}$ Here-returning to the point made by Jean-Philippe Nicolas-the number of perforations will be even: $0,2,4$ etc

${ }^{16}$ Deformations of $\partial \Omega$ can of course lead to the exclusion or inclusion of certain loops.
} 
But if I dwell on these old three-dimensional theories I may give the impression I want to make a point about them, whereas my real point concerns the Aharonov-Bohm effect: it is hardly topological, or at any rate no more topological than electrostatics etc.

I thank Nazim Bouatta, Dennis Dieks, Éric Gourgoulhon, Marc Lachièze-Rey and Jean-Philippe Nicolas for valuable clarifications and corrections.

\section{References}

Agricola, I. and T. Friedrich (2010) Vektoranalysis: Differentialformen in Analysis, Geometrie und Physik, Vieweg+Teubner, Berlin

Aharonov, Y. and D. Bohm (1959) "Significance of electromagnetic potentials in the quantum theory" Physical Review 115, 485-91

Batterman, R. W. (2003) "Falling cats, parallel parking and polarized light" Studies in History and Philosophy of Modern Physics 34, 527-57

Brading, K. and E. Castellani, editors (2003) Symmetries in physics, Cambridge University Press

Ehrenberg, W. and R. E. Siday (1949) "The refractive index in electron optics and the principles of dynamics" Proceedings of the Physical Society B 62, 8-21

Lyre, H. (2001) "The principles of gauging” Philosophy of Science 68, S371-81

Lyre, H. (2004) "Holism and structuralism in U(1) gauge theory" Studies in History and Philosophy of Modern Physics 35, 643-70

Martin, C. (2003) "On continuous symmetries and the foundations of modern physics" pp. 29-60 in Brading \& Castellani (2003)

Nash, C. and S. Sen (1983) Topology and geometry for physicists, Academic Press, London

Nounou, A. (2003) "A fourth way to the Aharonov-Bohm effect" pp. 174-99 in Brading \& Castellani (2003)

Ryder, L. (1996) Quantum field theory, Cambridge University Press

Wu, T. T. and C. N. Yang (1975) "Concept of nonintegrable phase factors and global formulation of gauge fields" Physical Review D 12, 3845-57 